\title{
RITAN: rapid integration of term annotation and network resources
}

\author{
Michael T Zimmermann ${ }^{1,2,3}$, Brian Kabat ${ }^{3}$, Diane E Grill ${ }^{3}$, Richard B Kennedy ${ }^{4}$, Gregory A Poland ${ }^{\text {Corresp. } 4}$ \\ 1 Bioinformatics Research and Development Laboratory, Genomic Sciences and Precision Medicine Center, Medical College of Wisconsin, Milwaukee, \\ Wisconsin, United States \\ 2 Clinical and Translational Sciences Institute, Medical College of Wisconsin, Milwaukee, Wisconsin, United States \\ 3 Division of Biomedical Statistics and Informatics, Department of Health Sciences Research, Mayo clinic, Rochester, Minnesota, United States \\ 4 Mayo Clinic Vaccine Research Group, Mayo Clinic, Rochester, Minnesota, United States \\ Corresponding Author: Gregory A Poland \\ Email address: poland.gregory@mayo.edu
}

Background. Identifying the biologic functions of groups of genes identified in highthroughput studies currently requires considerable time and/or bioinformatics experience. This is due in part to each resource housed within separate databases, requiring users to know about them, and integrate across them. Time consuming and often repeated for each study, integrating across resources and merging with data under study is an increasingly common bioinformatics task. Methods. We developed an open-source $\mathrm{R}$ software package for assisting researchers in annotating their genesets with functions, pathways, and their interconnectivity across a diversity of network resources. Results. We present RITAN for the rapid and comprehensive annotation of a list of genes using functional term and pathway resources and their relationships among each other using multiple network biology resources. Currently, and to comply with data redistribution policies, RITAN allows rapid access to 16 term annotations spanning GO, biologic pathways, and immunologic modules, and nine network biology resources, with support for user-supplied resources; we provide recommendations for additional resources and scripts to facilitate their addition to RITAN. Having the resources together in the same system allows users to derive novel combinations. RITAN has a growing set of tools to explore the relationships within resources themselves. These tools allow users to merge resources together such that the merged annotations have a minimal overlap with one another. Because we index both function annotation and network interactions, the combination allows users to expand small groups of genes using links from biologic networks - either by adding all neighboring genes or by identifying genes that efficiently connect among input genes - followed by term enrichment to identify functions. That is, users can start from a core set of genes, identify interacting genes from biologic networks, and then identify the functions to which the expanded list of genes contribute. Conclusion. We believe RITAN Peer) reviewing PDF | (2018:09:31074:1:0:NEW 1 Apr 2019) 
fills the important niche of bridging the results of high-throughput experiments with the ever-growing corpus of functional annotations and network biology resources.

Availability. RITAN is available as an R package at github.com/MTZimmer/RITAN and BioConductor.org. 
1 RITAN: Rapid Integration of Term Annotation and Network Resources 2

3 Michael T. Zimmermann ${ }^{1,2,3}$, Brian Kabat ${ }^{3}$, Diane E. Grill ${ }^{3}$, Richard B. Kennedy ${ }^{4}$, Gregory A.

4 Poland $^{4, *}$

5 'Bioinformatics Research and Development Laboratory, Genomics Sciences and Precision

6 Medicine Center, Medical College of Wisconsin, Milwaukee, WI 53226, USA

$7 \quad{ }^{2}$ Clinical and Translational Sciences Institute, Medical College of Wisconsin, Milwaukee, WI 8 53226, USA

9 'Division of Biomedical Statistics and Informatics, Department of Health Sciences Research, 10 Mayo Clinic, Rochester, MN USA

$11{ }^{4}$ Mayo Clinic Vaccine Research Group, Mayo Clinic, Rochester, MN USA

$12 *$ Corresponding author electronic address: poland.gregory@mayo.edu

13

14 Keywords: Gene Networks, systems biology, enrichment analysis, open-source software, 15 Software Tools, genomic data interpretation, knowledge generation 


\section{Abstract}

Background. Identifying the biologic functions of groups of genes identified in high-throughput studies currently requires considerable time and/or bioinformatics experience. This is due in part to each resource housed within separate databases, requiring users to know about them, and integrate across them. Time consuming and often repeated for each study, integrating across resources and merging with data under study is an increasingly common bioinformatics task. Methods. We developed an open-source R software package for assisting researchers in annotating their genesets with functions, pathways, and their interconnectivity across a diversity of network resources. Results. We present RITAN for the rapid and comprehensive annotation of a list of genes using functional term and pathway resources and their relationships among each other using multiple network biology resources. Currently, and to comply with data redistribution policies, RITAN allows rapid access to 16 term annotations spanning GO, biologic pathways, and immunologic modules, and nine network biology resources, with support for usersupplied resources; we provide recommendations for additional resources and scripts to facilitate their addition to RITAN. Having the resources together in the same system allows users to derive novel combinations. RITAN has a growing set of tools to explore the relationships within resources themselves. These tools allow users to merge resources together such that the merged annotations have a minimal overlap with one another. Because we index both function annotation and network interactions, the combination allows users to expand small groups of genes using links from biologic networks - either by adding all neighboring genes or by identifying genes that efficiently connect among input genes - followed by term enrichment to identify functions. That is, users can start from a core set of genes, identify interacting genes from biologic networks, and then identify the functions to which the expanded list of genes contribute. Conclusion. We believe RITAN fills the important niche of bridging the results of high-throughput experiments with the ever-growing corpus of functional annotations and network biology resources. Availability. RITAN is available as an R package at github.com/MTZimmer/RITAN and BioConductor.org.

45

46

47

\section{Introduction}

High-throughput technologies are enabling systems-level assays for an increasing diversity of applications, which is revealing previously unknown genetic underpinnings of many diseases and phenotypes. It is common for researchers to identify a list of potentially interesting genes, many of which have no known relationship to the condition studied. In this situation, it is challenging to identify the functions these genes contribute to, their precise mechanisms, and the role each gene plays in orchestrating those functions. Further, it is necessary to estimate how many of the prioritized genes may be chance associations due to biologic variability. Many data systems and computational tools have been developed to aid researchers in addressing these critical challenges; however, to better understand how genes interact with each other to achieve a common function, the comprehensive integration of multiple annotation and network biology resources typically remains a data wrangling venture re-invented for each research project. 
57 Many resources have been developed to standardize and codify terms or descriptive annotations

58 for the functions of genes or their encoded products. Ontologies such as GO (Ashburner et al.

59 2000) aim to describe each gene specifically and comprehensively. Pathway databases such as

60 PID (Schaefer et al. 2009), KEGG (Kanehisa et al. 2012), and others, gather sets of genes

61 participating in a particular function that has defined initiation points and outcomes and the

62 topology that connects them. A third approach is to identify which genes are co-regulated across

63 conditions (functional modules), as exemplified by Hallmarks in MSigDB (Subramanian et al.

64 2005) or blood transcription modules (Li et al. 2014). Resources like DisGeNet index

65 relationships between genes and diseases.(Pinero et al. 2015) Broadening the concept of a

66 pathway, network biology resources including HPRD (Prasad et al. 2009), CCSB (Rolland et al.

67 2014), and STRING (Szklarczyk et al. 2011) seek a more comprehensive view - how genes,

68 proteins, and potentially other molecules broadly interact with one another. Each of these

69 resource types has its own strengths and limitations. Therefore, in order to gain the most comprehensive understanding of how a group of genes relate to each other, and what biologic functions they may define or contribute to, data integration is necessary.

We have generated an open-source and publically available software package, RITAN, for the Rapid Integration of Term Annotation and Network resources. For term annotation or enrichment analysis, our system currently indexes 16 resources spanning GO, pathways, and immunologic modules, and facilitates annotation of a list of genes (e.g., those differentially expressed). Users may query one resource at a time or multiple resources together, allowing false discovery to be accounted for across all resources used. For network biology, nine resources are indexed and confidence-score filtering is enabled when available. See our vignettes within the package for the full list of resources. Users may add resources using standard file formats to extend the depth and breadth of coverage. By using these resources and adding additional resources of interest, analyses can be more efficient, standardized, and reproducible by minimizing manual steps and keeping resources indexed in a common and accessible system. We have used this integrated network resource in our previous studies (Haralambieva et al. 2016a; Haralambieva et al. 2016b; Kennedy et al. 2016; Zimmermann et al. 2016) to provide an organizational framework to unify results from multiple omics data (mRNA, protein, DNA methylation), yielding an integrated signature that is more informative than each alone.

\section{Methods}

RITAN is implemented as a package in the $\mathrm{R}$ programming language. It leverages multiple existing packages, extending their utility, including igraph (Csardi \& Nepusz 2006) and STRINGdb. Enrichment analysis currently uses the hypergeometric test.

Reproducible analysis examples are available in package vignettes, which are also available in our supplemental data. We also downloaded normalized breast invasive carcinoma (BRCA) mRNA-Seq gene expression data from the Cancer Genome Atlas (TCGA) pan-cancer analysis

94 (Ding et al. 2018) for demonstrating selected utilities. We used EdgeR (McCarthy et al. 2012) to 
98 We have developed a self-contained executable R-Shiny application to bring some of RITAN's

99 functionality to a broader audience. The app leverages a combination of technologies including

100 the chromium web browser, electron stand-alone server, and RPortable, and built into a self-

101 contained Windows executable using node.js.

\section{Results}

103 RITAN is an enabling tool for identifying the functional associations within datasets generated

104 by high-throughput methods. We have implemented features that help researchers to uncover

105 relationships within their dataset and augmented by closely related genes in biologic networks

106 (Figure 1A and 1B). We used a study of anti-TREM1 effects on influenza response, as an

107 example (Figure 1C and 1D). Data for this study is publicly posted on GEO under the accession

108 ID, GSE9988 (Dower et al. 2008). The study considered multiple treatments and lists of genes

109 associated with each comparison of those treatments were published. Using RITAN, multiple

110 resources were combined into a single enrichment analysis, allowing more comprehensive false-

111 discovery control. The visualization made by RITAN shows a study design matrix with

112 enrichment heatmap to visually annotate how different treatments activate different responses

113 (Figure 1C). Differential pathway activity by each treatment is presented visually and

114 numerically. Genes associating with up-regulated responses are further contextualized by

115 identification of their known molecular inter-relationships (Figure 1D). The network information

116 can be directly mined, used in computation, or visualized. In this case, we have uploaded the

117 integrated network returned by RITAN to Cytoscape for visualization and scaled each gene by

118 the number of interactions they have as an indication of how "central" they are to response. The

119 network facilitates hypothesis generation by visually displaying the differential association with

120 anti-TREM1 response with different NFkB subunits, differential Akt regulation via JUN, PTEN,

121 GRB2, etc., and different neutrophil chemotactic signals (e.g. FPR3 or CXCL3). Thus, the

122 network is a hypothesis generating tool, focused by first identifying the most relevant subset of

123 results from our integrated enrichment analysis. Through data within biologic networks, genes

124 that were not differentially expressed, but closely interact with differentially expressed genes,

125 can be identified, implicating their potential activity change despite no mRNA expression

126 change.

127

128 In addition to facilitating rapid functional annotation, RITAN includes tools to analyze the

129 relationships between resources. For example, many diseases are contributed to by similar sets of

130 genes (Figure 2A). Additionally, many diseases are characterized by alteration of specific

131 biologic processes or molecular functions (Figure 2B). By identifying how resources overlap

132 (fraction of shared genes), we are able to combine the resources in a way that reduces the

133 overlap. Once reduced to a set of terms or pathways that are more independent, enrichment

134 analysis can be run on the combined set of terms and using a more accurate false discovery

135 adjustment. Without reducing overlapping terms, false discovery adjustment across resources

136 will be overly conservative. Additionally, having many resources in the same system allows

137 exploration of relationships among them, and making novel combinations.

138 We use RITAN as a simple knowledge management system that facilitates data annotation and

139 hypothesis exploration - activities that are nor supported by other tools or are challenging to use 
140 programmatically. As additional demonstrations of the utility enabled by RITAN, we

141 investigated differential gene expression profiles between breast cancer subtypes from TCGA.

142 This analysis is to demonstrate an approach to data annotation and exploration enabled by

143 RITAN, rather than a definitive analysis of this specific and well-studied dataset. While we

144 distribute many resources with RITAN, other resource licenses prohibit redistribution. We

145 provide recommendations for additional resources (see our GitHub page; we plan to implement

146 an automated download and setup script). Our use of simple and accepted file formats simplifies

147 the process of adding resources. Leveraging additional resources, we annotated the differentially

148 expressed genes between subtypes, first identifying which of them are shared between specific

149 subtypes, part of known protein complexes, define rare diseases, or have clearly defined

150 regulatory programs (Figure 3A). Multiple protein complexes have differentially expressed

151 genes between disease subtypes including SNARE and the chromosomal passenger complex.

152 Both of these complexes have known roles in carcinogenesis and breast cancer.(Kabisch et al.

153 2015; Meng \& Wang 2015) Multiple rare disease genes are differentially expressed and

154 contribute to precursor B- and T-cell acute lymphoblastic leukemia, among others. Next, we

155 considered Gene Ontology term (Figure 3B) and Reactome pathway (Figure 3C) enrichment

156 among genes differentially expressed between disease subtypes, and for the further subset of

157 differentially expressed genes that contribute to rare diseases. These annotations highlight the

158 role of metabolic differences among disease subtypes and that these functional overlap rare

159 disease mechanisms, complementing the use of rare disease annotations for prioritizing

160 functional somatic variants.(Ma et al. 2017) Finally, we compared GO terms to Reactome

161 pathways to identify which were shared and which were unique. There are $5231 \mathrm{GO}$ terms with a

162 one-to-one mapping to 1607 Reactome pathways (100\% of genes shared), and 83 GO terms that

163 highly overlap 244 Reactome pathways (95\% of genes shared; Figure 3D). Further vignettes are

164 available within the package and are included in our Supplemental Data. These analyses among

165 gene annotation resources facilitate hypothesis generation and exploration.

\section{Discussion}

167 Enrichment analysis has become commonplace in bioinformatics and biostatistics; however,

168

169

170

171

172

173

174

175

176

177

178

179

180

181 current tools have limitations. First, most resources are distributed independently of each other and integrating across them is left to individual users. Second, each enrichment or pathway analysis is typically performed independently, preventing false discovery adjustment across all tests performed. Further, while web servers are convenient for small numbers of queries, programmatic solutions are an advantage for data analysis workflows and scripting for reproducible research.

Enrichment analysis is a statistical method wherein an input list of genes is compared to a term, function, or pathway definition. The definition is usually a pre-existing list of genes curated to describe a specific cellular function or observation. The number of genes in the pathway is recorded and combined with the size of the input list, the size of the term or pathway, and the total number of genes that were assayed (e.g. all human protein coding genes expressed in the tissue of interest), an overrepresentation statistic is computed. When this statistic is significant (e.g. occurring with a probability less than 0.01 by chance and after false discovery correction), the term is typically ascribed to the input list and we describe the function as "enriched for" 
182 within the input list. Thus, defining all three areas is critical: the input list, the term definitions, 183 and the universe of possible gene results.

184 We encourage users to decide upon an analysis strategy prior to running their analysis, including

185

186

187

188

189

190

191

192

193

194

195

196

197

198

199

200

201

202

203

204

205

206

207

208

209

210

211

212

213

214

215

216

217

218

219

220 consideration of which resources are most appropriate for their dataset/experiment, statistical significance thresholds, background geneset, etc. The ease with which RITAN facilitates multiresource query may lead users to "try one more test," leading to an increased number of hypothesis tests made that may not be accounted for by multiple testing correction. To prevent this and appropriately maintain false-discovery correction, we encourage users to "include one more resource" - to add an additional resource to a single query in RITAN.

Other tools that fill similar roles to RITAN exist, but each within a specific niche, using specific data sources, or having additional input requirements. Within the R framework, GAGE (Luo et al. 2009) provides access to GO and KEGG database for multiple species. Reactome offers related services, but annotations are specific to their database. Web servers for enrichment analysis have been developed including WebGestalt (Wang et al. 2013) and DAVID (Huang da et al. 2009). The flexibility of RITAN and ability to integrate and explore relationships among both geneset and network resources, distinguishes RITAN from existing software.

RITAN allows annotation integration across many publically available resources; thus, it facilitates rapid development of novel hypotheses about the potential functions achieved by prioritized genes and multiple-testing correction across all resources used. The annotations from RITAN can be leveraged by existing down-stream analysis tools, such as Cytoscape (Shannon et al. 2003) or igraph (Csardi \& Nepusz 2006), in order to provide greater visualization and network-analytic power. As illustrated in Figure 1, the two major features of RITAN are an integrated system for term enrichment and connections among genes via network biology, facilitating reproducible research and hypothesis generation. For analyses across resources, it is important to consider how similar the resources are to each other. Similarities may occur within a pathway resource or even between resource types. For example, within the KEGG pathway resource, JAK/STAT and MAPK signaling share SOS, RAS, RAF, and other genes. Ontology, pathway, and network information can be applied to multiple entities: gene-centric, proteincentric, or term-centric. For example, the GO term GO:1901184 marks the process "regulation of ERBB signaling pathway" and genes are annotated with this term when they are involved in the process, while KEGG's hsa04012 pathway indexes the relationships between genes participating in the "ERBB Signaling Pathway." In this particular case, the GO term is conceptually the same as the pathway, but without topology. Ontologies aim for greater specificity for each gene while pathways aim for greater resolution on a particular process or function. If similar terms, such as the GO term for ERBB signaling and the KEGG pathway for ERBB signaling, are included in the same analysis, false-discovery correction may be more stringent than is needed. Thus, functions within RITAN to identify, and filter resources base on semi-redundant or highly overlapping concepts, will assist the interpretation of data derived from high-throughput sequencing.

We also distinguish between a discovery p-value and an annotation p-value. For instance, when calculating differentially expressed genes, a discovery p-value is generated and is one of the

Peer] reviewing PDF | (2018:09:31074:1:0:NEW 1 Apr 2019) 
223

224

225

226

227

228

229

230

231

232

233

234

235

236

237

238

239

240

241

242

243

244

245

246

247

248

249

250

251

252

253

254

255

256

257

258

259

260

261

262

263

264

criteria for calling a gene differentially expressed. False-discovery or other statistical controls should be used at this stage. After discovering differentially expressed genes, RITAN can be a powerful tool to assist interpreting those results. The p-values generated in annotation have a different interpretation and role from discovery. Researchers should always be aware of weather they are using a statistical test for discovery (inference) or annotation. Iterating between discovery and annotation should be strictly avoided or explicitly identified - iterating can be a powerful discovery approach, but it can quickly lead to over-fitting. Statistical frameworks for combined feature selection and classification exist and could be adapted to implement an iterative approach in this setting.

We have active plans to expand RITAN's capabilities by adding additional statistical testing methods (Subramanian et al. 2005), supporting improved resource-reduction methods, and novel metrics for the statistical significance of network overlaps (Ding et al. 2016; Zimmermann et al. 2016). Non-programmers could benefit from the capabilities of RITAN. Thus, we have developed a self-contained executable application and made the app available for download on RITAN's github page. We plan to further develop both resources - to improve the core features of RITAN and expand our portable application for use by a broader audience. The features currently within RITAN are highly useful and their implementation in a common framework facilitates ease of use and reproducible research.

\section{Conclusion}

RITAN fills a niche in being a simple gene-centric knowledge management system - to index geneset and network resources - as well as provide multiple tools for resource integration, assessment, enrichment, and exploration. We believe RITAN will be an enabling tool for the interpretation of high-throughput genomic studies.

\section{Acknowledgements}

The authors acknowledge support from NIH grant U01AI089859 for this work and thank Caroline L. Vitse for her editorial assistance.

\section{References}

R Core Team. R: A Language and Environment for Statistical Computing. R Foundation for Statistical Computing (http://www.R-project.org). 2014.

Ashburner M, Ball CA, Blake JA, Botstein D, Butler H, Cherry JM, Davis AP, Dolinski K, Dwight SS, Eppig JT, Harris MA, Hill DP, Issel-Tarver L, Kasarskis A, Lewis S, Matese JC, Richardson JE, Ringwald M, Rubin GM, and Sherlock G. 2000. Gene ontology: tool for the unification of biology. The Gene Ontology Consortium. Nature genetics 25:25-29. $10.1038 / 75556$

Barabasi AL, Gulbahce N, and Loscalzo J. 2011. Network medicine: a network-based approach to human disease. Nature reviews Genetics 12:56-68. 10.1038/nrg2918

Cerami EG, Gross BE, Demir E, Rodchenkov I, Babur O, Anwar N, Schultz N, Bader GD, and Sander C. 2011. Pathway Commons, a web resource for biological pathway data. Nucleic acids research 39:D685-690. 10.1093/nar/gkq1039

Croft D, O'Kelly G, Wu G, Haw R, Gillespie M, Matthews L, Caudy M, Garapati P, Gopinath G, Jassal B, Jupe S, Kalatskaya I, Mahajan S, May B, Ndegwa N, Schmidt E, Shamovsky V, Yung C, Birney E, Hermjakob H, D'Eustachio P, and Stein L. 2011. Reactome: a 
265

266

267

268

269

270

271

272

273

274

275

276

277

278

279

280

281

282

283

284

285

286

287

288

289

290

291

292

293

294

295

296

297

298

299

300

301

302

303

304

305

306

307

308

309

310

311

312

313

314

315

database of reactions, pathways and biological processes. Nucleic acids research 39:D691-697. 10.1093/nar/gkq1018

Csardi G, and Nepusz T. 2006. The igraph software package for complex network research. InterJournal Complex Systems:1695.

Ding L, Bailey MH, Porta-Pardo E, Thorsson V, Colaprico A, Bertrand D, Gibbs DL, Weerasinghe A, Huang KL, Tokheim C, Cortes-Ciriano I, Jayasinghe R, Chen F, Yu L, Sun S, Olsen C, Kim J, Taylor AM, Cherniack AD, Akbani R, Suphavilai C, Nagarajan N, Stuart JM, Mills GB, Wyczalkowski MA, Vincent BG, Hutter CM, Zenklusen JC, Hoadley KA, Wendl MC, Shmulevich L, Lazar AJ, Wheeler DA, Getz G, and Cancer Genome Atlas Research N. 2018. Perspective on Oncogenic Processes at the End of the Beginning of Cancer Genomics. Cell 173:305-320 e310. 10.1016/j.cell.2018.03.033

Ding Z, Zhang X, Sun D, and Luo B. 2016. Overlapping Community Detection based on Network Decomposition. Scientific reports 6:24115. 10.1038/srep24115

Dower K, Ellis DK, Saraf K, Jelinsky SA, and Lin LL. 2008. Innate immune responses to TREM1 activation: overlap, divergence, and positive and negative cross-talk with bacterial lipopolysaccharide. Journal of immunology 180:3520-3534.

Haralambieva IH, Ovsyannikova IG, Kennedy RB, Zimmermann MT, Grill DE, Oberg AL, and Poland GA. 2016a. Transcriptional signatures of influenza A/H1N1-specific IgG memorylike B cell response in older individuals. Vaccine 34:3993-4002. 10.1016/j.vaccine.2016.06.034

Haralambieva IH, Zimmermann MT, Ovsyannikova IG, Grill DE, Oberg AL, Kennedy RB, and Poland GA. 2016b. Whole Transcriptome Profiling Identifies CD93 and Other Plasma Cell Survival Factor Genes Associated with Measles-Specific Antibody Response after Vaccination. PloS one 11:e0160970. 10.1371/journal.pone.0160970

Huang da W, Sherman BT, and Lempicki RA. 2009. Systematic and integrative analysis of large gene lists using DAVID bioinformatics resources. Nature protocols 4:44-57. 10.1038/nprot.2008.211

INSERM. Orphanet: an online database of rare diseases and orphan drugs. Copyright, INSERM 1997. Available at http://www.orpha.net.

Kabisch M, Lorenzo Bermejo J, Dunnebier T, Ying S, Michailidou K, Bolla MK, Wang Q, Dennis J, Shah M, Perkins BJ, Czene K, Darabi H, Eriksson M, Bojesen SE, Nordestgaard BG, Nielsen SF, Flyger H, Lambrechts D, Neven P, Peeters S, Weltens C, Couch FJ, Olson JE, Wang X, Purrington K, Chang-Claude J, Rudolph A, Seibold P, Flesch-Janys D, Peto J, dos-Santos-Silva I, Johnson N, Fletcher O, Nevanlinna H, Muranen TA, Aittomaki K, Blomqvist C, Schmidt MK, Broeks A, Cornelissen S, Hogervorst FB, Li J, Brand JS, Humphreys K, Guenel P, Truong T, Menegaux F, Sanchez M, Burwinkel B, Marme F, Yang R, Bugert P, Gonzalez-Neira A, Benitez J, Pilar Zamora M, Arias Perez JI, Cox A, Cross SS, Reed MW, Andrulis IL, Knight JA, Glendon G, Tchatchou S, Sawyer EJ, Tomlinson I, Kerin MJ, Miller N, kConFab I, Australian Ovarian Cancer Study G, Haiman CA, Schumacher F, Henderson BE, Le Marchand L, Lindblom A, Margolin S, Hooning MJ, Hollestelle A, Kriege M, Koppert LB, Hopper JL, Southey MC, Tsimiklis H, Apicella C, Slettedahl S, Toland AE, Vachon C, Yannoukakos D, Giles GG, Milne RL, McLean C, Fasching PA, Ruebner M, Ekici AB, Beckmann MW, Brenner H, Dieffenbach AK, Arndt V, Stegmaier C, Ashworth A, Orr N, Schoemaker MJ, Swerdlow A, GarciaClosas M, Figueroa J, Chanock SJ, Lissowska J, Goldberg MS, Labreche F, Dumont M, Winqvist R, Pylkas K, Jukkola-Vuorinen A, Grip M, Brauch H, Bruning T, Ko YD, Network G, Radice P, Peterlongo P, Scuvera G, Fortuzzi S, Bogdanova N, Dork T, Mannermaa A, Kataja V, Kosma VM, Hartikainen JM, Devilee P, Tollenaar RA, Seynaeve C, Van Asperen CJ, Jakubowska A, Lubinski J, Jaworska-Bieniek K, Durda K, Zheng W, Shrubsole MJ, Cai Q, Torres D, Anton-Culver H, Kristensen V, Bacot F, Tessier DC, Vincent D, Luccarini C, Baynes C, Ahmed S, Maranian M, Simard J, 
316

317

318

319

320

321

322

323

324

325

326

327

328

329

330

331

332

333

334

335

336

337

338

339

340

341

342

343

344

345

346

347

348

349

350

351

352

353

354

355

356

357

358

359

360

361

362

363

364

365

366
Chenevix-Trench G, Hall P, Pharoah PD, Dunning AM, Easton DF, and Hamann U.

2015. Inherited variants in the inner centromere protein (INCENP) gene of the chromosomal passenger complex contribute to the susceptibility of ER-negative breast cancer. Carcinogenesis 36:256-271. 10.1093/carcin/bgu326

Kandasamy K, Mohan SS, Raju R, Keerthikumar S, Kumar GS, Venugopal AK, Telikicherla D, Navarro JD, Mathivanan S, Pecquet C, Gollapudi SK, Tattikota SG, Mohan S, Padhukasahasram H, Subbannayya Y, Goel R, Jacob HK, Zhong J, Sekhar R, Nanjappa V, Balakrishnan L, Subbaiah R, Ramachandra YL, Rahiman BA, Prasad TS, Lin JX, Houtman JC, Desiderio S, Renauld JC, Constantinescu SN, Ohara O, Hirano T, Kubo M, Singh S, Khatri P, Draghici S, Bader GD, Sander C, Leonard WJ, and Pandey A. 2010. NetPath: a public resource of curated signal transduction pathways. Genome biology 11:R3. 10.1186/gb-2010-11-1-r3

Kanehisa M, and Goto S. 2000. KEGG: kyoto encyclopedia of genes and genomes. Nucleic acids research 28:27-30.

Kanehisa M, Goto S, Sato Y, Furumichi M, and Tanabe M. 2012. KEGG for integration and interpretation of large-scale molecular data sets. Nucleic acids research 40:D109-114. 10.1093/nar/gkr988

Kennedy RB, Ovsyannikova IG, Haralambieva IH, Oberg AL, Zimmermann MT, Grill DE, and Poland GA. 2016. Immunosenescence-Related Transcriptomic and Immunologic Changes in Older Individuals Following Influenza Vaccination. Front Immunol 7:450. 10.3389/fimmu.2016.00450

Li S, Rouphael N, Duraisingham S, Romero-Steiner S, Presnell S, Davis C, Schmidt DS, Johnson SE, Milton A, Rajam G, Kasturi S, Carlone GM, Quinn C, Chaussabel D, Palucka AK, Mulligan MJ, Ahmed R, Stephens DS, Nakaya HI, and Pulendran B. 2014. Molecular signatures of antibody responses derived from a systems biology study of five human vaccines. Nature immunology 15:195-204. 10.1038/ni.2789

Loscalzo J. 2011. Systems biology and personalized medicine: a network approach to human disease. Proceedings of the American Thoracic Society 8:196-198. 10.1513/pats.201006-041MS

Luo W, Friedman MS, Shedden K, Hankenson KD, and Woolf PJ. 2009. GAGE: generally applicable gene set enrichment for pathway analysis. BMC bioinformatics 10:161. 10.1186/1471-2105-10-161

Ma M, Wang C, Glicksberg BS, Schadt EE, Li SD, and Chen R. 2017. Identify Cancer Driver Genes through Shared Mendelian Disease Pathogenic Variants and Cancer Somatic Mutations. Pac Symp Biocomput 22:473-484. 10.1142/9789813207813_0044

McCarthy DJ, Chen Y, and Smyth GK. 2012. Differential expression analysis of multifactor RNA-Seq experiments with respect to biological variation. Nucleic acids research 40:4288-4297. 10.1093/nar/gks042

Meng J, and Wang J. 2015. Role of SNARE proteins in tumourigenesis and their potential as targets for novel anti-cancer therapeutics. Biochimica et biophysica acta 1856:1-12. 10.1016/j.bbcan.2015.04.002

Mi H, Muruganujan A, Casagrande JT, and Thomas PD. 2013. Large-scale gene function analysis with the PANTHER classification system. Nature protocols 8:1551-1566. 10.1038/nprot.2013.092

Pinero J, Queralt-Rosinach N, Bravo A, Deu-Pons J, Bauer-Mehren A, Baron M, Sanz F, and Furlong LI. 2015. DisGeNET: a discovery platform for the dynamical exploration of human diseases and their genes. Database : the journal of biological databases and curation 2015:bav028. 10.1093/database/bav028

Prasad TS, Kandasamy K, and Pandey A. 2009. Human Protein Reference Database and Human Proteinpedia as discovery tools for systems biology. Methods in molecular biology 577:67-79. 10.1007/978-1-60761-232-2_6 
367

368

369

370

371

372

373

374

375

376

377

378

379

380

381

382

383

384

385

386

387

388

389

390

391

392

393

394

395

396

397

398

399

400

401

402

403

404

405

406

407

408

409

410

411

412

413

414

Rolland T, Tasan M, Charloteaux B, Pevzner SJ, Zhong Q, Sahni N, Yi S, Lemmens I, Fontanillo C, Mosca R, Kamburov A, Ghiassian SD, Yang X, Ghamsari L, Balcha D, Begg BE, Braun P, Brehme M, Broly MP, Carvunis AR, Convery-Zupan D, Corominas R, Coulombe-Huntington J, Dann E, Dreze M, Dricot A, Fan C, Franzosa E, Gebreab F, Gutierrez BJ, Hardy MF, Jin M, Kang S, Kiros R, Lin GN, Luck K, MacWilliams A, Menche J, Murray RR, Palagi A, Poulin MM, Rambout X, Rasla J, Reichert P, Romero V, Ruyssinck E, Sahalie JM, Scholz A, Shah AA, Sharma A, Shen Y, Spirohn K, Tam S, Tejeda AO, Trigg SA, Twizere JC, Vega K, Walsh J, Cusick ME, Xia Y, Barabasi AL, lakoucheva LM, Aloy P, De Las Rivas J, Tavernier J, Calderwood MA, Hill DE, Hao T, Roth FP, and Vidal M. 2014. A proteome-scale map of the human interactome network. Cell 159:1212-1226. 10.1016/j.cell.2014.10.050

Ruepp A, Waegele B, Lechner M, Brauner B, Dunger-Kaltenbach I, Fobo G, Frishman G, Montrone C, and Mewes HW. 2010. CORUM: the comprehensive resource of mammalian protein complexes--2009. Nucleic acids research 38:D497-501. 10.1093/nar/gkp914

Schaefer CF, Anthony K, Krupa S, Buchoff J, Day M, Hannay T, and Buetow KH. 2009. PID: the Pathway Interaction Database. Nucleic acids research 37:D674-679. 10.1093/nar/gkn653

Shannon P, Markiel A, Ozier O, Baliga NS, Wang JT, Ramage D, Amin N, Schwikowski B, and Ideker T. 2003. Cytoscape: a software environment for integrated models of biomolecular interaction networks. Genome research 13:2498-2504. 10.1101/gr. 1239303

Subramanian A, Tamayo P, Mootha VK, Mukherjee S, Ebert BL, Gillette MA, Paulovich A, Pomeroy SL, Golub TR, Lander ES, and Mesirov JP. 2005. Gene set enrichment analysis: a knowledge-based approach for interpreting genome-wide expression profiles. Proceedings of the National Academy of Sciences of the United States of America 102:15545-15550. 10.1073/pnas.0506580102

Szklarczyk D, Franceschini A, Kuhn M, Simonovic M, Roth A, Minguez P, Doerks T, Stark M, Muller J, Bork P, Jensen LJ, and von Mering C. 2011. The STRING database in 2011: functional interaction networks of proteins, globally integrated and scored. Nucleic acids research 39:D561-568. 10.1093/nar/gkq973

Vinayagam A, StelzI U, Foulle R, Plassmann S, Zenkner M, Timm J, Assmus HE, AndradeNavarro MA, and Wanker EE. 2011. A directed protein interaction network for investigating intracellular signal transduction. Science signaling 4:rs8. 10.1126/scisignal.2001699

Wang J, Duncan D, Shi Z, and Zhang B. 2013. WEB-based GEne SeT AnaLysis Toolkit (WebGestalt): update 2013. Nucleic acids research 41:W77-83. 10.1093/nar/gkt439 Zimmermann MT, Oberg AL, Grill DE, Ovsyannikova IG, Haralambieva IH, Kennedy RB, and Poland GA. 2016. System-Wide Associations between DNA-Methylation, Gene Expression, and Humoral Immune Response to Influenza Vaccination. PloS one $11: \mathrm{e} 0152034$. 10.1371/journal.pone.0152034

Figure 1: RITAN facilitates rapid and comprehensive annotation and network integration. We arbitrarily chose GSE9988 (Dower et al. 2008), a study of anti-TREM1 effects on influenza response, as an example. (A) From an input gene list (blue nodes), RITAN leverages multiple network resources to identify neighbors (green nodes) and (B) performs integrated term enrichment. (C) Using RITAN, multiple resources were combined into a single enrichment analysis with results presented as a heatmap where greater intensity indicates greater statistical 
415 significance. The values in the heatmap are the $-\log _{10}(\mathrm{q}$-value) with values capped at 10 for

416 visualization. Adding a study design matrix above the enrichment heatmap (also by RITAN)

417 visually annotates how each treatment lead to different pathway enrichment that capture aspects

418 of inflammation, innate immunity, cytokine signaling, and interferon signaling. Considering the

419 two comparisons emphasized by black borders, LPS \& anti-TREM1-antibody versus control,

420 many terms and pathways exhibiting significant differences in enrichment. (D) RITAN also

421 integrates across multiple network biology resources. The genes associating with up-regulated

422 responses are further contextualized by identification of their known inter-relationships. We used

423 Cytoscape to visualize the integrated network returned by RITAN. We have scaled each gene's

424 size by the number of interactions they have. Differential association with anti-TREM1 response

425 are indicated by red outline.

426

427 Figure 2: RITAN facilitates comparing resource similarities. (A) Within the DisGeNet 428 resource, we compare the overlap of genes annotated to each disease. A disease is shown if it 429 shares at least an $80 \%$ overlap with another disease, ignoring self-overlap. (B) Between two 430 resources, DisGeNet and Go Slim, we show interactions among terms when any pair shares at 431 least $95 \%$ overlap. Term and disease names are too small for print legibility, but can be viewed 432 when zoomed in on the electronic version.

433

Figure 3: RITAN facilitates hypothesis generation and exploration. We used TCGA BRCA differential gene expression data and RITAN to explore specific hypotheses. (A) We annotated differentially expressed genes among luminal A (LumA), Basal, and HER2+ subtypes by their presence in protein complexes, rare disease definitions, and known regulatory programs. The overlapping genes for each combination of annotations indicates additional hypotheses to explore. We used a 5-way Venn diagram to show how many genes have each annotation. (B) An example of an additional hypothesis is how functional term enrichment may differ, not only between disease subtypes, but also by contribution to rare disease definitions. We show enrichment plots by subgroup formatted similar to Figure 1. (C) Additionally, how pathway activation may differ. (D) Similar to Figure 2, RITAN can be used to explore relationships among resources and to filter each resource to a unique subset. The analysis script for this figure is short (93 lines including comments and plotting; available in Supplemental Data), 


\section{Figure 1}

RITAN facilitates rapid and comprehensive annotation and network integration.

We use the arbitrarily chosen example of GSE9988, a study of anti-TREM1 effects on influenza response. This study considered multiple treatments and lists of genes associated with each comparison of those treatments have been published. (A) From an input gene list (blue nodes), RITAN leverages multiple network resources to identify neighbors (green nodes) and (B) performs integrated term enrichment. We use GSE9988 (Dower et al. 2008), a study of anti-TREM1 effects on influenza response, as an example. Using RITAN, multiple resources were combined into a single enrichment analysis with results presented as a heatmap where greater intensity indicates greater statistical significance. The values in the heatmap are the - $\log _{10}(q-v a l u e)$ with values capped at 10 for visualization. To better use the color scale. Adding a study design matrix above the enrichment heatmap (also by RITAN) visually reveals that significant enrichment scores are defined by the up-regulated gene lists and that they capture aspects of inflammation, innate immunity, cytokine signaling, and interferon signaling. Considering the two comparisons emphasized by a black border, LPS \& anti-TREM1-antibody versus control-treated, there are many terms and pathways exhibiting significant differences in enrichment. (C) RITAN also integrates across multiple network biology resources. The genes associating with up-regulated responses in panel $B$ are further contextualized by identification of their known inter-relationships. The network information can be directly mined, used in computation, or visualized. In this case, we have uploaded the integrated network returned by RITAN to Cytoscape for visualization. We have scaled each gene by the number of interactions they have as an indication of how "central" they are to response. The network facilitates hypothesis generation by visually displaying the differential association with anti-TREM1 response (indicated by red outline) with different NFkB subunits, 
differential Akt regulation via JUN, PTEN, GRB2, etc., and different neutrophil chemotactic signals (e.g. FPR3 or CXCL3). Thus, the network is a hypothesis generating tool, focused by first identifying the most relevant subset of results from our integrated enrichment analysis. (D) RITAN integrates network biology resources, which can be directly visualized or imported into Cytoscape. In this example, JUN, NFKB1, TNF, and multiple cytokines were not differentially expressed, but their interactions with the differentially expressed genes were identified, implicating their activity in cellular responses.
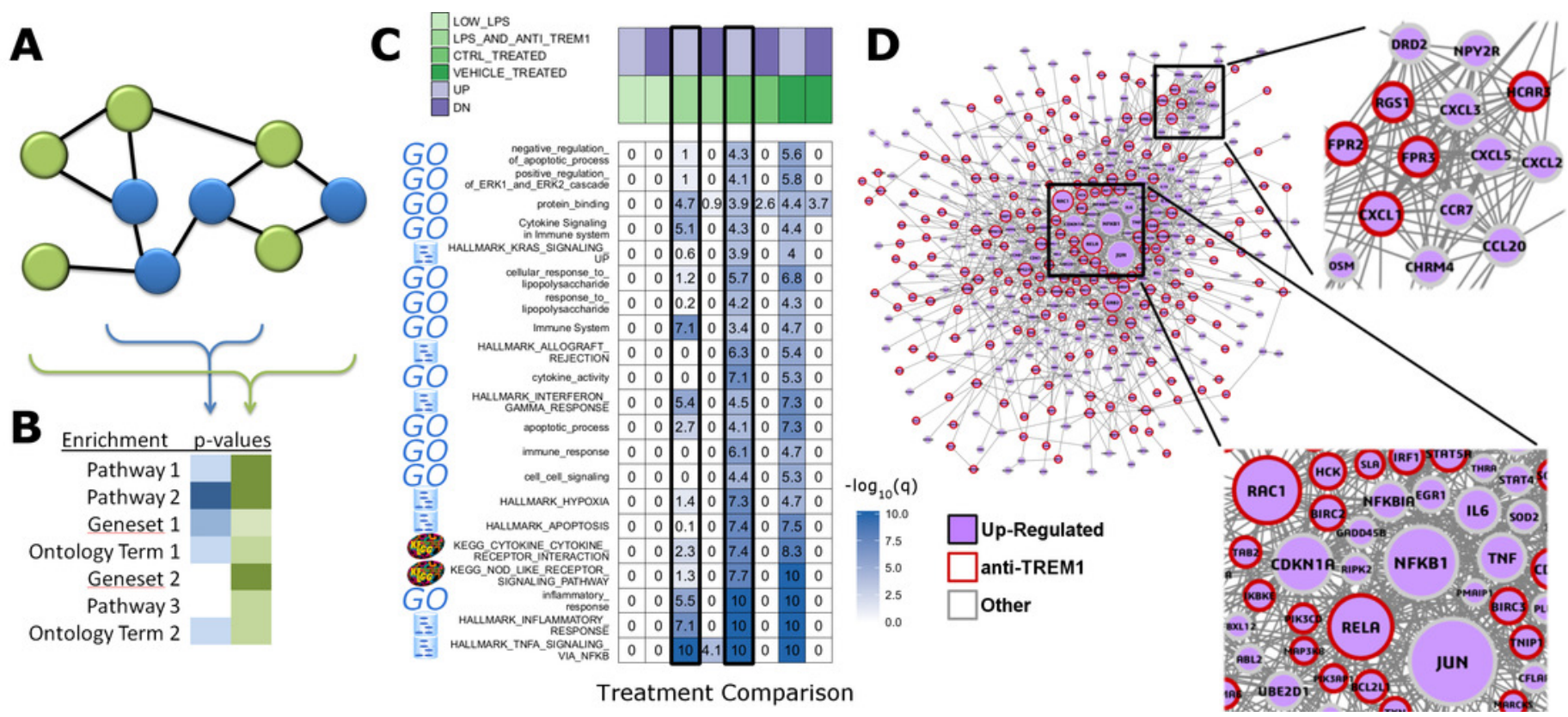


\section{Figure 2 (on next page)}

RITAN facilitates comparing resource similarities.

(A) Within the DisGeNet resource, we compare the overlap of genes annotated to each disease. A disease is shown if it shares at least an $80 \%$ overlap with another disease, ignoring self-overlap. (B) Between two resources, DisGeNet and Go Slim, we show interactions among terms when any pair shares at least $95 \%$ overlap. 


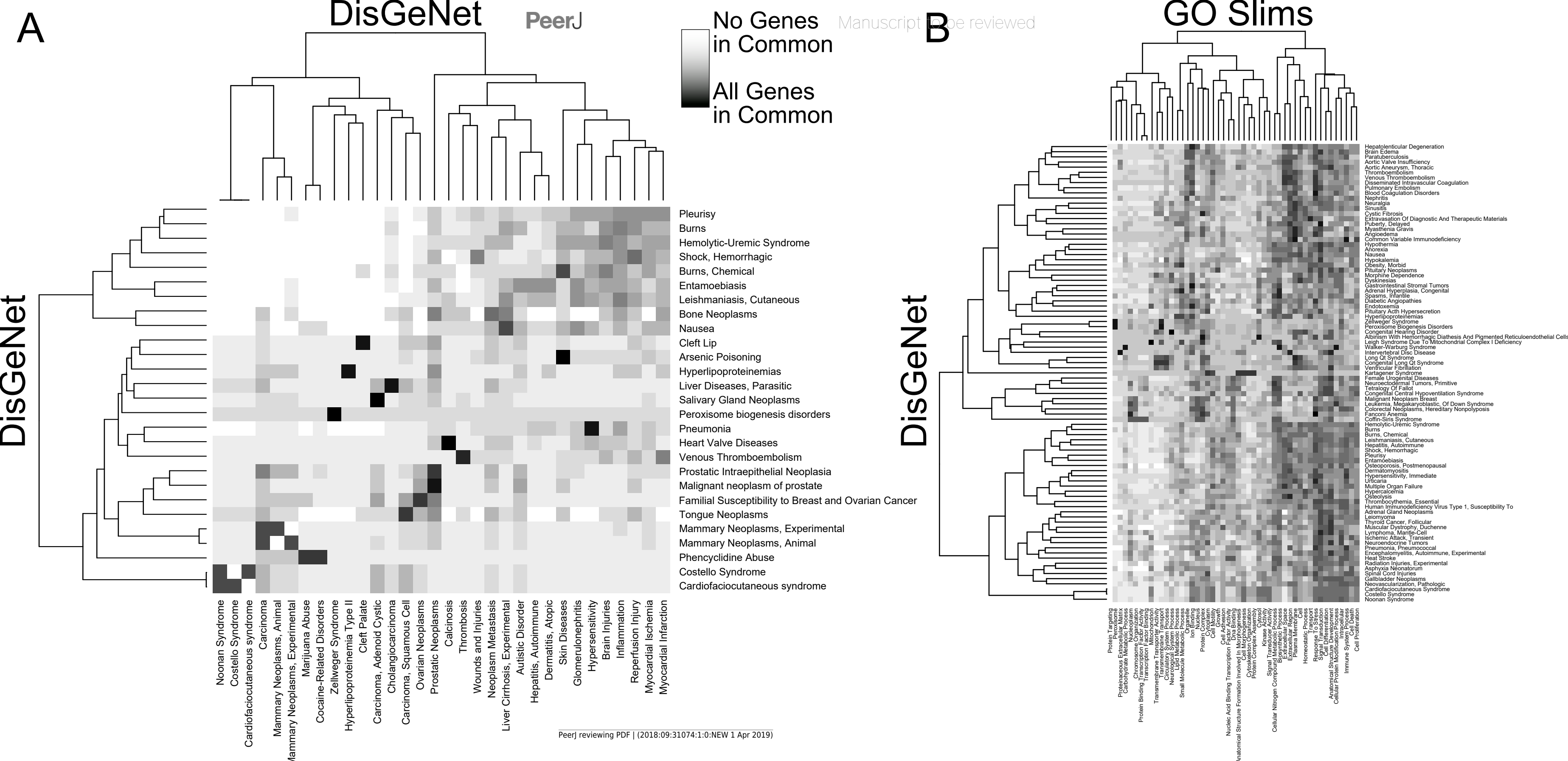




\section{Figure 3 (on next page)}

RITAN facilitates hypothesis generation and exploration

We used TCGA BRCA differential gene expression data and RITAN to explore specific hypotheses. (A) We annotated differentially expressed genes among luminal A (LumA), Basal, and HER2 + subtypes by their presence in protein complexes, rare disease definitions, and known regulatory programs. The overlapping genes for each combination of annotations indicates additional hypotheses to explore. We used a 5-way Venn diagram to show how many genes have each annotation. (B) An example of an additional hypothesis is how functional term enrichment may differ, not only between disease subtypes, but also by contribution to rare disease definitions. We show enrichment plots by subgroup formatted similar to Figure 1. (C) Additionally, how pathway activation may differ. (D) Similar to Figure 2, RITAN can be used to explore relationships among resources and to filter each resource to a unique subset. The analysis script for this figure is short (93 lines including comments and plotting; available in Supplemental Data), emphasizing the flexibility and simplicity afforded by RITAN. 


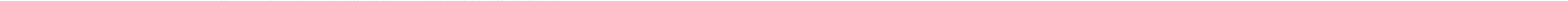

